\title{
Distúrbios vocais associados ao AVC: uma revisão sistemática
}

\author{
Vocal disorders associated with stroke: a systematic review
}

\author{
Trastornos vocales asociados con el ACV: \\ una revisión sistemática
}

\author{
Beatriz Vitorio Ymai Rosendo실 Laura Faustino Gonçalves ${ }^{2}$, \\ Karina Mary De Paiva ${ }^{3}$, Patrícia Haas ${ }^{4}$
}

\begin{abstract}
1.Universidade Federal de Santa Catarina - UFSC; Departamento de Fonoaudiologia. Florianópolis-SC, Brasil. ORCID: https://orcid.org/0000-0002-7485-2797

2.Universidade Federal de Santa Catarina - UFSC; Departamento de Fonoaudiologia. Florianópolis-SC, Brasil. ORCID: https://orcid.org/0000-0002-0043-4349

3.Universidade Federal de Santa Catarina - UFSC; Departamento de Fonoaudiologia. Florianópolis-SC, Brasil. ORCID: https://orcid.org/0000-0001-7086-534X

4.Universidade Federal de Santa Catarina - UFSC; Departamento de Fonoaudiologia. Florianópolis-SC, Brasil. ORCID: https://orcid.org/0000-0001-9797-7755
\end{abstract}

\begin{abstract}
Resumo
Introdução. A voz é primordial para os seres humanos e depende da integridade das estruturas neurológicas e anatômicas relacionadas à fonação. Dentre as implicações do AVC associadas com a voz, os distúrbios vocais afetam diretamente a inteligibilidade da fala e a saúde vocal dos indivíduos. Objetivo. Apresentar evidências científicas com base em revisão sistemática da literatura (PRISMA) respondendo à seguinte pergunta norteadora: Quais os possíveis distúrbios vocais associados ao AVC? Método. A busca de artigos foi realizada nas bases de dados Scielo, Lilacs, Pubmed, Scopus, Bireme e Web Of Science, não houve restrição de localização, período e idioma. Para a seleção dos estudos foi utilizada a combinação baseada no Medical Subject Heading Terms (MeSH). Para complementar, foi realizada uma busca por literatura cinza no Google Scholar. Foram admitidos na pesquisa dois estudos que obtiveram pontuação $\geq 6$ pontos segundo o protocolo para pontuação qualitativa proposto por Pithon et al. (2015). Foram identificados 181 artigos com potencial para inclusão, sendo dois correspondentes aos critérios de inclusão definidos e à pergunta norteadora. Resultados. Os sujeitos acometidos pelo AVC apresentaram aspectos de vozes rugosas, soprosas, instáveis e pastosas, às vezes tensas, além de lentidão e instabilidade no controle motor laríngeo. Não houve associação direta do comportamento vocal ao AVC com a topografia da lesão encefálica. Conclusão. Dentre os distúrbios vocais associados ao AVC, identificou-se a classificação regular da disartria espástica e flácida, seguida de aspectos vocais como a rugosidade, soprosidade, tensão vocal exagerada, instabilidade na emissão de vogais e velocidade reduzida.
\end{abstract}

Unitermos. Acidente Vascular Cerebral; Distúrbios da Voz; Voz; Fonoaudiologia

\begin{abstract}
Introduction. The voice is essential for human beings and depends on the integrity of the neurological and anatomical structures related to phonation. Among the implications of stroke associated with the voice, vocal disorders directly affect speech intelligibility and vocal health of individuals. Objective. To present scientific evidence based on a systematic literature review (PRISMA) answering the following guiding question: What are the possible vocal disorders associated with stroke? Method. The search for articles was carried out in the databases Scielo, Lilacs, Pubmed, Scopus, Bireme and Web of Science, there was no restriction on location, period and language. For the selection of studies, the combination based on the Medical Subject Heading Terms (MeSH) was used. In addition, a search for gray literature was carried out on Google Scholar. Two studies that scored $\geq 6$ points according to the qualitative scoring protocol proposed by Pithon et al. (2015). 181 articles with potential for inclusion were identified, two corresponding to the defined inclusion criteria and the guiding question. Results. The subjects affected by the stroke presented aspects of rough, breathy, unstable,
\end{abstract}


and pasty voices, sometimes tense, in addition to slowness and instability in laryngeal motor control. There was no direct association between vocal behavior and stroke with the topography of the brain injury. Conclusion. Among the vocal disorders associated with stroke, the regular classification of spastic and flaccid dysarthria was identified, followed by vocal aspects such as roughness, breathiness, exaggerated vocal tension, instability in the emission of vowels and reduced speed.

Keywords. Stroke; Voice Disorders; Voice; Speech Therapy

\section{RESUMEN}

Introducción. La voz es fundamental para el ser humano y depende de la integridad de las estructuras neurológicas y anatómicas relacionadas con la fonación. Entre las implicaciones del accidente cerebrovascular asociado con la voz, los trastornos vocales afectan directamente la inteligibilidad del habla y la salud vocal de los individuos. Objetivo. Presentar evidencia científica basada en una revisión sistemática de la literatura (PRISMA) respondiendo a la siguiente pregunta orientadora: ¿Cuáles son los posibles trastornos vocales asociados al ictus? Método. La búsqueda de artículos se realizó en las bases de datos Scielo, Lilacs, Pubmed, Scopus, Bireme y Web Of Science, sin restricciones de ubicación, época o idioma. Para la selección de estudios, se utilizó una combinación basada en los Términos de Encabezamiento de Materia Médica (MeSH). Además, se realizó una búsqueda en Google Scholar de literatura gris. En la investigación se incluyeron dos estudios que obtuvieron una puntuación $\geq 6$ puntos según el protocolo de puntuación cualitativa propuesto por Pithon et al. (2015). Se identificaron 181 artículos con potencial de inclusión, dos correspondientes a los criterios de inclusión definidos y la pregunta orientadora. Resultados. Los sujetos afectados por ACV presentaron aspectos de voces ásperas, entrecortadas, inestables y pastosas, a veces tensas, además de lentitud e inestabilidad en el control motor laríngeo. No hubo asociación directa entre el comportamiento vocal del accidente cerebrovascular y la topografía de la lesión cerebral. Conclusión. Entre los trastornos vocales asociados al ictus, se identificó la clasificación regular de disartria espástica y flácida, seguida de aspectos vocales como aspereza, disnea, tensión vocal exagerada, inestabilidad en la emisión de vocales y velocidad reducida.

Palabras clave: Accidente cerebrovascular; Trastornos de la voz; Voz; Terapia del lenguaje

Trabalho realizado na Universidade Federal de Santa Catarina - UFSC. Florianópolis-SC, Brasil.

\section{INTRODUÇÃO}

As doenças cardiovasculares (DCV) representam a principal causa de mortalidade no Brasil desde os anos 60, sendo em alguns centros urbanos a principal causa desde os anos 401. Estas correspondem a um grupo de lesões do Sistema Nervoso Central (SNC), assim incluindo o Acidente Vascular Cerebral (AVC), cujos danos cerebrais decorrem do déficit no suprimento neuronal ${ }^{2}$. De acordo com a Organização Mundial de Saúde (OMS) ${ }^{3}$, o AVC é uma das 
complicações cardiovasculares predominantes no mundo, e se trata da principal causa de incapacidade funcional ${ }^{4}$. As complicações decorrentes do AVC interferem significativamente na qualidade de vida do indivíduo; uma vez que as sequelas podem comprometer os mecanismos responsáveis por funções primordiais como a fala, voz, deglutição e respiração ${ }^{5-8}$. No Brasil, 30\% a $48 \%$ dos pacientes sobreviventes do AVC apresentam alguma forma de incapacidade, limitando significativamente o desempenho funcional das pessoas, com consequências nas relações pessoais, familiares e, sobretudo, na qualidade de vida9,10.

Dentre as implicações do AVC envolvidas com voz, as disartrias são distúrbios vocais que acompanham de $20 \%$ a $40 \%$ das lesões cerebrais, sendo caracterizadas por alterações motoras da respiração, fonação, articulação, ressonância e prosódia, e tais características interferem na inteligibilidade da fala ${ }^{11,12}$. Além disso, as lesões neurológicas podem afetar intensamente as funções laríngeas e faríngeas, acometendo, consequentemente, a saúde vocal do indivíduo ${ }^{13}$.

As alterações vocais podem evoluir em diferentes graus, consequentemente afetando a qualidade de vida em diferentes níveis ${ }^{14}$. A voz é primordial para os seres humanos e depende de estruturas neurológicas e anatômicas relacionadas à fonação íntegras ${ }^{15}$ essenciais para a expressão dos pensamentos, emoções, e para o convívio social. A partir do exposto, a presente pesquisa determina como objetivo principal e norteador verificar evidências 
científicas sobre os possíveis distúrbios vocais associados ao AVC, visando responder a seguinte pergunta norteadora de pesquisa: Quais os possíveis distúrbios vocais associados ao AVC?

\section{MÉTODO}

A presente revisão sistemática foi conduzida conforme as recomendações PRISMA (Preferred Reporting Items for Systematic reviews and Meta-Analyses) ${ }^{16}$. As buscas por artigos científicos foram conduzidas por dois pesquisadores independentes nas bases de dados eletrônicas MEDLINE (Pubmed), LILACS, SciELO, SCOPUS, WEB OF SCIENCE e BIREME, sem restrição de idioma, período e localização. A pesquisa foi estruturada e organizada na forma PICOS (Tabela 1), que representa um acrônimo para População alvo, Intervenção, Comparação e "Outcomes" (desfechos). População de interesse ou problema de saúde corresponde à pacientes; intervenção (I): distúrbios vocais; comparação (C): AVC; outcome (O): alteração da voz (S): estudo transversal, estudo observacional, relatos de caso, estudos de caso-controle, ensaios clínicos controlados, estudos de coorte.

\section{Estratégia de pesquisa}

Os descritores foram selecionados a partir do dicionário Descritores em Ciências da Saúde (DeCS) e Medical Subject Heading Terms (MeSH), haja vista a sua grande utilização 
pela comunidade científica para a indexação de artigos na base de dados PubMed.

Tabela 1. Descrição dos componentes do PICOS.

\begin{tabular}{cl}
\hline Acrônimo & Definição \\
\hline $\mathbf{P}$ & Pacientes \\
\hline $\mathbf{I}$ & Distúrbios Vocais \\
\hline $\mathbf{C}$ & AVC \\
\hline $\mathbf{O}$ & Alteração da voz \\
\hline $\mathbf{S}$ & Estudo transversal \\
& Estudo observacional \\
& Relatos de caso \\
& Estudos de caso-controle \\
& Ensaios clínicos controlados \\
& Estudos de coorte \\
\hline
\end{tabular}

Diante da busca dos descritores, foi realizada a adequação para as outras bases utilizadas. Em um primeiro momento foram propostas para as buscas os seguintes descritores e operador booleano: (cerebral vascular accident) and (vocal disorders) and (voice). A busca ocorreu de forma concentrada em novembro de 2020. Para complementar, foi realizada uma busca por literatura cinza no Google Scholar. 


\section{Critérios de elegibilidade}

Os desenhos do estudo selecionado poderiam ser do tipo descritivo, transversal, observacional, de caso-controle, de coorte, ensaios clínicos controlados, relatos de caso estudo e estudo qualitativo. Foram inclusos estudos sem restrição de idioma, período e localização. A Tabela 2 representa os critérios de inclusão e exclusão desenvolvidos nesta pesquisa. Os estudos obtiveram pontuação 12 no protocolo modificado ${ }^{17}$ para avaliação da qualidade.

Tabela 2. Síntese dos critérios de inclusão/exclusão.

\begin{tabular}{ll}
\hline Critérios de Inclusão \\
\hline Delineamento & $\begin{array}{l}\text { Relatos de casos } \\
\text { Estudos de casos e controle } \\
\text { Ensaios clínicos controlados } \\
\text { Estudos de coorte } \\
\text { Estudos em triagem } \\
\text { Estudos observacionais }\end{array}$ \\
\hline Localização & Sem Restrição \\
\hline Idioma & Sem restrição \\
\hline Critérios de Exclusão \\
\hline Delineamento & $\begin{array}{l}\text { Cartas ao editor } \\
\text { Diretrizes } \\
\text { Revisões de literatura } \\
\text { Revisões sistemáticas } \\
\text { Meta-análises }\end{array}$ \\
\hline Estudos & $\begin{array}{l}\text { Estudos pouco } \\
\text { inadequados }\end{array}$ \\
\hline $\begin{array}{l}\text { Forma } \\
\text { de publicação }\end{array}$ & Apenas resumo \\
\hline
\end{tabular}

\section{Risco de viés}

A qualidade dos métodos foi avaliada pelos revisores de forma independente (KMP e $\mathrm{PH}$ ), de acordo com a 
recomendação PRISMA ${ }^{16}$. A avaliação priorizou a descrição clara das informações. Neste ponto, a revisão foi realizada às cegas, mascarando os nomes dos autores e revistas, evitando qualquer viés potencial e conflito de interesses.

\section{Critérios de exclusão}

Foram excluídos estudos publicados no formato de Cartas ao editor, diretrizes, revisões de literatura, revisões sistemáticas, meta análises e resumos. Estudos que foram pouco claros ou, ainda, indisponíveis na íntegra (Tabela 2).

\section{Análise dos dados}

A extração dos dados para o processo de elegibilidade dos estudos foi realizada utilizando-se uma ficha própria para revisão sistemática elaborada por dois pesquisadores em Programa Excel ${ }^{\circledR}$, na qual os dados extraídos foram adicionados por um dos pesquisadores e, então, conferidos por outro pesquisador. Inicialmente foram selecionados de acordo com o título; em seguida, os resumos foram analisados e apenas os que fossem potencialmente elegíveis foram selecionados. Com base nos resumos, artigos foram selecionados para leitura integral, foram admitidos os que atendiam a todos os critérios pré-determinados. Em caso de desacordo entre avaliadores, um terceiro avaliador (KMP) tomou a decisão sobre a elegibilidade do estudo em questão. 


\section{Forma de seleção dos estudos}

Inicialmente os revisores de elegibilidade ( $\mathrm{PH}$ e KMP) foram calibrados para a realização da revisão sistemática por BVYR e LFG. Após a calibração e esclarecimentos de dúvidas, os títulos e resumos foram examinados pelos revisores de elegibilidade (KMP e PH), de forma independente, os quais não estavam cegos para o nome dos autores e das revistas. Aqueles que apresentaram um título dentro do âmbito, mas os resumos não estavam disponíveis, também foram obtidos e analisados na íntegra. Foram excluídos estudos fora do escopo proposto, relatos de caso, cartas ao editor e/ou editorial, revisões de literatura, índices, resumos e estudos em animais. Posteriormente, os estudos elegíveis preliminarmente tiveram o texto completo obtido e avaliado. Em casos específicos, quando o estudo com potencial de elegibilidade apresentasse dados incompletos, os autores poderiam ser contatados por e-mail para mais informações, no entanto não foi necessário este contato.

\section{Dados coletados}

Após a triagem, o texto do artigo selecionado foi revisado e extraído de forma padronizada por dois autores (BVYR e LFG) sob a supervisão de KMP e PH, identificandose ano de publicação, local da pesquisa, idioma de publicação, tipo de estudo, amostra, método, resultado e conclusão do estudo. 


\section{Resultado clínico}

O resultado clínico de interesse foi verificar evidências científicas sobre os possíveis distúrbios vocais associados ao AVC. Aqueles que não utilizaram esta abordagem específica e pré-determinada, não fizeram parte da amostra admitida para a revisão de literatura.

\section{RESULTADOS}

Inicialmente foram selecionados 181 artigos, dimensionados para 167 após exclusão por repetição; em seguida, os títulos e resumos foram analisados e 165 trabalhos foram excluídos, pois não estavam no escopo da proposta da pesquisa. Assim, admitiram-se dois artigos para a análise final ${ }^{18,20}$, ambos estudos de relato de caso (Figura $1)$.

Figura 1. Fluxograma de busca e análise dos artigos.
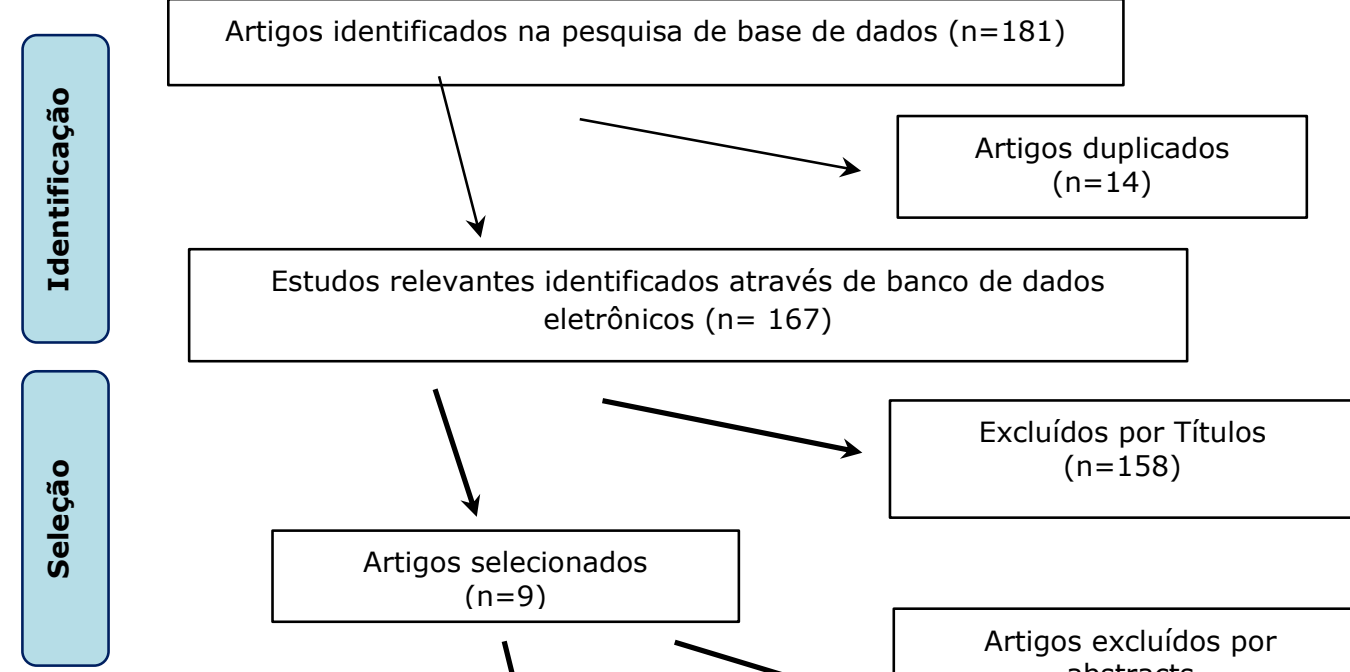

Artigos selecionados $(n=9)$
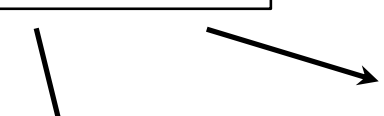

Artigos excluídos por abstracts $(n=7)$

$$
(n=2)
$$


A partir dos descritores elêitos, os bancos de dados foram consultados e foram obtidos os resultados disponibilizados na Tabela 3.

Tabela 3. Classificação das referências obtidas nas bases de dados Pubmed, Scielo, Lilacs, Web of Science e Scopus.

\begin{tabular}{|c|c|c|c|c|c|}
\hline Descritores & No & $\begin{array}{l}\text { Referências } \\
\text { excluídas }\end{array}$ & Motivo & Selecionado & Banco de dados \\
\hline $\begin{array}{l}\text { (cerebral vascular } \\
\text { accident) and } \\
\text { (vocal disorders) } \\
\text { and (voice) }\end{array}$ & 156 & 155 & $\begin{array}{l}\text { Excluídos por repetição (9); } \\
\text { excluídos por título (4); } \\
\text { excluídos por resumo (142) }\end{array}$ & 1 & Pubmed \\
\hline $\begin{array}{l}\text { (cerebral vascular } \\
\text { accident) and } \\
\text { (vocal disorders) } \\
\text { and (voice) }\end{array}$ & 3 & 2 & $\begin{array}{l}\text { Excluído por resumo (1); } \\
\text { excluídos por título (2) }\end{array}$ & 1 & Lilacs \\
\hline $\begin{array}{l}\text { (cerebral vascular } \\
\text { accident) and } \\
\text { (vocal disorders) } \\
\text { and (voice) }\end{array}$ & 0 & - & - & - & Scielo \\
\hline $\begin{array}{l}\text { (cerebral vascular } \\
\text { accident) and } \\
\text { (vocal disorders) } \\
\text { and (voice) }\end{array}$ & 0 & - & - & - & WEB OF SCIENCE \\
\hline $\begin{array}{l}\text { (cerebral vascular } \\
\text { accident) and } \\
\text { (vocal disorders) } \\
\text { and voice)(cerebral } \\
\text { vascular accident) } \\
\text { and (vocal } \\
\text { disorders) and } \\
\text { (voice) }\end{array}$ & 22 & 22 & $\begin{array}{l}\text { Excluídos por repetição (5); } \\
\text { excluídos por resumo (15); } \\
\quad \text { excluídos por título (2) }\end{array}$ & 0 & Bireme \\
\hline $\begin{array}{l}\text { (cerebral vascular } \\
\text { accident) and } \\
\text { (vocal disorders) } \\
\text { and (voice) }\end{array}$ & 0 & - & - & - & SCOPUS \\
\hline Total & 181 & 179 & & 2 & Pubmed e Lilacs \\
\hline
\end{tabular}

Com relação à descrição dos resultados dos artigos elegíveis neste estudo, as informações podem ser verificadas detalhadamente na Tabela 4. Os métodos utilizados, bem como resultados e conclusão das pesquisas se encontram expostos na referida tabela. Os achados dos estudos incluídos na presente análise referentes ao comprometimento da voz pós-AVC se fundamentam em 


\section{análises acústicas da fonação mediante avaliações}

perceptivo-auditivas.

Tabela 4. Síntese dos artigos incluídos.

\begin{tabular}{|c|c|c|c|c|c|}
\hline $\begin{array}{l}\text { Autor/ } \\
\text { Ano/ Local } \\
\text { de } \\
\text { publicação }\end{array}$ & Objetivo & $\begin{array}{c}\text { N da } \\
\text { amostra }\end{array}$ & Método & Resultados & Conclusão \\
\hline $\begin{array}{l}\text { Wang et al., } \\
2009^{20} \\
\text { China }\end{array}$ & $\begin{array}{l}\text { Relatar a análise } \\
\text { acústica } \\
\text { fonação da vogal } \\
\text { sustentada em } \\
\text { indivíduos com } \\
\text { disartria pós-AVC, } \\
\text { comparando com } \\
\text { dados normativos } \\
\text { para idosos e } \\
\text { jovens saudáveis }\end{array}$ & $\begin{array}{l}25 \\
\text { mulheres; } \\
63 \text { homens }\end{array}$ & $\begin{array}{l}\text { A amostra do discurso } \\
\text { foram duas tentativas de } \\
\text { prolongamento da vogal } \\
\text { /a/. Foram selecionados } \\
15 \text { indicadores acústicos } \\
\text { do MDVP e utilizados } \\
\text { dados normativos de } \\
\text { indivíduos com } \\
\text { envelhecimento } \\
\text { saudável. A classificação } \\
\text { da disartria foi realizada } \\
\text { por um fonoaudiólogo. } \\
\text { Análise acústica } \\
\text { realizada pelo MDVP, e o } \\
\text { software SPSS } 14.0 \\
\text { comparou os parâmetros } \\
\text { entre os grupos } \\
\text { (indivíduos com AVC, } \\
\text { idosos saudáveis, jovens } \\
\text { saudáveis) }\end{array}$ & $\begin{array}{lr}\text { A maior parte } & \text { dos } \\
\text { participantes } & \text { foram } \\
\text { classificados } & \text { com } \\
\text { disartria do neurónio } \\
\text { motor } & \text { superior } \\
\text { unilateral. Os homens } \\
\text { com AVC apresentaram } \\
\text { SPI maior que os } \\
\text { homens jovens } \\
\text { saudáveis. As mulheres } \\
\text { com AVC apontaram } \\
\text { maior ruído no SPI do } \\
\text { que as idosas } \\
\text { saudáveis, além de } \\
\text { maior sPPQ e SPI do } \\
\text { que as jovens } \\
\text { saudáveis. }\end{array}$ & 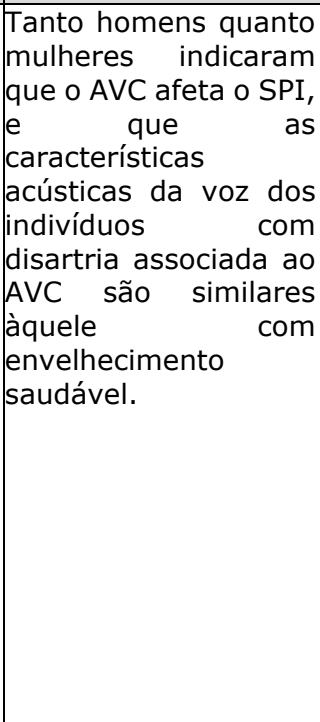 \\
\hline $\begin{array}{l}\text { Godoy et } \\
\text { al., } \\
2014^{18} \\
\text { Brasil }\end{array}$ & 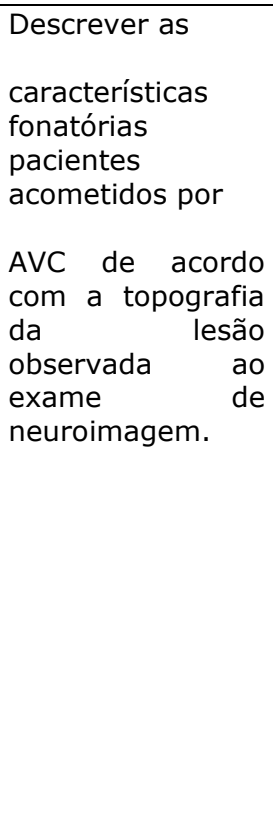 & $\begin{array}{l}6 \text { mulheres } \\
\text { e } 4 \text { homens }\end{array}$ & $\begin{array}{l}\text { Avaliação perceptivo- } \\
\text { auditiva da voz feita pelo } \\
\text { CAPE-V. Avaliação da } \\
\text { DDC por repetição da } \\
\text { vogal /a/ e /i/. Análise } \\
\text { das emissões realizada } \\
\text { pelo MSP, com } \\
\text { parâmetros para a DDC. } \\
\text { Análise dos dados foi } \\
\text { descritiva }\end{array}$ & $\begin{array}{l}\text { A maior parte } \\
\text { amostra indicou } \\
\text { extenso. Todos os } \\
\text { indivíduos } \\
\text { apresentaram algum } \\
\text { grau de soprosidade e } \\
\text { rugosidade na voz, e } \\
\text { metade tinha algum } \\
\text { grau de tensão vocal. A } \\
\text { prova de DDC relatou } \\
\text { lentificação r e } \\
\text { instabilidade } \\
\text { emissões. }\end{array}$ & 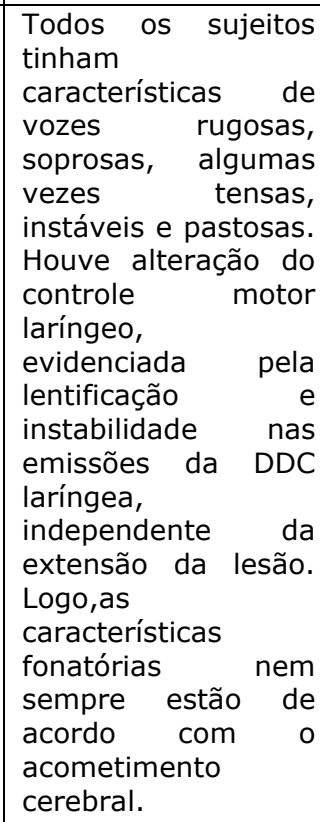 \\
\hline
\end{tabular}

AVC (Acidente Vascular Cerebral); MDVP (Programa Multi-Dimensional de Voz); SPI (índice de fonação suave); sPPQ (Quociente de perturbação do Pitch suavizado); CAPE-V (Consensus Auditory-Perceptual Evaluation of Voice); MSP (Motor Speech Profile Advanced); DDC (Diadococinesia). 
O estudo de Godoy $2014^{18}$ utilizou o protocolo CAPE-V (Consensus Auditory-Perceptual Evaluation of Voice) ${ }^{19}$, ferramenta para a avaliação vocal, desenvolvida por fonoaudiólogos integrantes da equipe americana Special Interest Division 3, Voice as Voice Disorder da American Speech-Language And Hearing Association (SID-3 - ASHA). Enquanto, Wang $2009^{20}$ adotaram o MDVP (Programa MultiDimensional de $\mathrm{Voz})^{19}$, programa de análise vocal oferecido pelo laboratório de Análise da Fala Computorizada da Kay Elemetrics Corp. A amostra da análise de Godoy $2014^{18}$ foi dividida em dois grupos de acordo com a TC dos indivíduos; um grupo com AVC de longa extensão e lesão na artéria cerebral média, e outro com AVC de menor extensão e lesão em localizações variadas do cérebro. Dentre estes, houve prevalência da disartria do neurônio motor superior unilateral (UUMN), mas foi representada também a disartria espástica. Ademais, em ambos os grupos, as anormalidades fonatórias referentes à emissão da vogal sustentada, fala e controle motor laríngeo foram independentemente associadas à topografia da lesão observada ao exame (extensão do AVC) ${ }^{18}$.

Utilizou-se o instrumento CAPE-V na descrição das características do comportamento vocal de indivíduos com mais de 60 anos acometidos por AVC - confirmado por tomografia computadorizada (TC) de crânio. Para tal, foram avaliados 6 parâmetros de qualidade vocal pré-determinados nas três tarefas: vogal prolongada, frases balanceadas e fala espontânea, bem como analisaram-se os aspectos de grau 
global da alteração vocal, rugosidade, soprosidade, tensão, pitch, loudness, instabilidade na emissão da vogal sustentada e pastosidade. A análise das emissões das vogais /a/ e /i/ ocorreu através do programa Motor Speech Profile Advanced (MSP), que forneceu os parâmetros para a diadococinesia laríngea (DDC). A partir disso, relatou-se que todos os indivíduos da amostra $(n=10)$ manifestaram pelo menos algum grau de soprosidade, rugosidade na voz, instabilidade na emissão da vogal sustentada e pastosidade durante a fala, tendo metade destes inclusive algum grau de tensão vocal. Houve também alteração no controle motor laríngeo, evidenciada pela lentificação e instabilidade nas emissões da DDC laríngea ${ }^{18}$.

Ademais, Wang $2009^{20}$ relatou a análise acústica da fonação específica da vogal /a/ sustentada em uma amostra de 88 indivíduos com disartria diagnosticada pós-AVC, e comparou os resultados com dados normativos de idosos e jovens saudáveis. Nesse caso, foram selecionados 15 parâmetros acústicos do MDVP. Assim, os achados evidenciaram parâmetros referentes ao índice de fonação suave (SPI) e ao coeficiente de perturbação do pitch suavizado ( $\mathrm{SPPQ}$ ) à medida que os indivíduos do sexo masculino com AVC revelaram padrão de parâmetros acústicos vocais similar aos homens idosos saudáveis, e identificou-se apenas o índice de fonação suave (SPI) significativamente menor nos homens com AVC. Os falantes do sexo feminino com AVC não apontaram diferenças significativas no SPPQ e SPI em relação às idosas com 
envelhecimento saudável, porém relataram maior SPPQ e SPI do que as mulheres jovens saudáveis. Destarte, os impactos do envelhecimento saudável sobre a mudança acústica da voz corresponderam, em sua maioria, aos efeitos do AVC $^{20}$.

\section{DISCUSSÃO}

As disfonias são distúrbios vocais presentes em casos de lesões neurológicas, sendo regulares em pacientes acometidos pelo $\mathrm{AVC}^{21}$. Em específico, a disartria é o distúrbio neuromotor representante das alterações vocais pós AVC, sendo avaliada por percepção auditiva conforme os parâmetros vocais: soprosidade (percepção de escape respiratório durante a fonação; rugosidade (percepção de irregularidades vocais pelas vibrações aperiódicas das PPVV); tensão (percepção de hiperfunção glótica); pitch (classificação da frequência fundamental da voz); loundness (correlação perceptual da intensidade sonora) ${ }^{22}$.

Nesse sentido, a partir da análise dos estudos compilados, pode se constatar em indivíduos acometidos pelo AVC aspectos vocais de rugosidade, soprosidade, e por vezes tensão vocal, bem como instabilidade durante a emissão da vogal /a/ sustentada, pastosidade ao longo da fala somada a lentidão e instabilidade no controle motor laríngeo ${ }^{20}$, como averiguado por outros autores ${ }^{23-26}$. Além disso, observou-se nos casos de AVC valores de grau geral elevado para as avaliações perceptivo-auditivas, 
especialmente no aspecto da vogal /a/ sustentada ${ }^{18}$, corroborando com outra pesquisa ${ }^{27}$. Indivíduos após 0 derrame indicaram atributos acústicos da voz durante o prolongamento da vogal similares aos de indivíduos mais velhos e saudáveis, porém há uma maior propensão de vozes ruidosas, com maior grau de desvio vocal nos sujeitos com $A_{V C}{ }^{20}$. Tal fato confirma a teoria de variação de frequência e adução das PVs em falantes saudáveis ${ }^{28}$, visto que 0 envelhecimento normal afeta também a função laríngea ${ }^{29}$.

Dentre os métodos para avaliação da qualidade vocal aplicados nos estudos incluídos, a análise acústica vocal ${ }^{20}$ é um instrumento que viabiliza uma descrição objetiva da disfunção da voz na fala disártrica, sendo apropriado e comumente utilizado para analisar a fonação da vogal sustentada30,31. Já, o protocolo para a avaliação perceptivoauditiva $^{18}$ oferece uma descrição do sinal vocal tendo como instrumento básico a audição ${ }^{32}$, e inclusive é muito importante para complementar o entendimento nos casos das disfagias orofaríngeas ${ }^{33}$. Por último, a DDC oral e laríngea são testes recomendados na avaliação da coordenação motora e a velocidade do movimento dos órgãos fonoarticulatórios em pacientes neurológicos ${ }^{34,35}$. De acordo com dados da literatura, a DDC laríngea é mais lenta em casos de alterações neurológicas ${ }^{36}$, tal como verificou Godoy $2014^{18}$.

Os distúrbios neurológicos da fala podem ser definidos de acordo com o nível anatômico afetado, sendo as lesões cerebrais vasculares parte dos transtornos do cerebelo ${ }^{37}$. 
Todavia, na associação entre o comportamento vocal e a topografia da lesão do AVC explorada pelo estudo brasileiro ${ }^{18}$, e por outros autores ${ }^{27}$, não se verificaram características vocais específicas de acordo com 0 acometimento cerebral. Entretanto, tanto os achados perceptivos vocais quanto da DDC oral são associados aos tipos de disartrias, e estas logo, são possivelmente caracterizadas quanto ao local da lesão no SNC. A exemplo disso, pacientes podem ser diagnosticados quanto à disartria, considerando a respiração, fonação, tarefas motoras orais, articulação, prosódia e inteligibilidade de fala ${ }^{23}$. Godoy $2014^{18}$ classificou a maior parte da amostra com disartria espástica em função das características fonatórias de pitch reduzido, rugosidade, voz tensa, quebras de frequência, frases curtas e velocidade reduzida em provas como a DDC. Da mesma forma, outro estudo brasileiro averiguou nos casos de AVC a prevalência da disartria espástica - distúrbio causado pela lesão no neurônio motor superior - e nesses casos os autores notaram ciclos respiratórios curtos, voz rouca, hipernasal, alteração moderada a grave de articulação, discreta alteração de prosódia e velocidade lenta de fala ${ }^{24}$.

Assim, dentre os artigos que referem o local da lesão encefálica ao acometimento do AVC, relatou-se que a disartria está associada a lesões tanto na região do telencéfalo e diencéfalo, quanto na região infratentorial ${ }^{38}$ sendo mais recorrente no hemisfério esquerdo. Foram abordados os impactos das disfonias e disartrias, e 
constataram alterações vocais mínimas no AVC de alcance unilateral ${ }^{39}$, enquanto o AVC bilateral causa disartrias mais severas, com lesão do neurônio motor superior ${ }^{38}$.

\section{CONCLUSÃO}

As lesões neurológicas decorrentes do AVC resultam em significativos comprometimentos na voz. Embora não haja relação específica das características do comportamento vocal após o AVC em função do local e extensão do acometimento encefálico, identificou-se a periódica classificação da disartria espástica e flácida, seguida de aspectos vocais como: rugosidade, soprosidade, tensão vocal exagerada, instabilidade na emissão de vogais e velocidade reduzida. Os pacientes neurológicos, em função das lesões ou alterações no SNC, estão sujeitos a alterações vocais, e a consequente evolução destas ${ }^{13}$. Portanto, independentemente do local e extensão da lesão neuronal, ou faixa etária, é evidente o impacto dos distúrbios da voz na qualidade de vida do indivíduo. Logo, destaca-se a relevância das avaliações e os métodos de reabilitação funcional dos pacientes acometidos pelo AVC.

\section{REFERÊNCIAS}

1. Laurenti R, Fonseca LA. A mortalidade por doença cardiovascular no Município de São Paulo em um período de 30 anos (1940-69). Arq Bras Cardiol 1976;29:855-8.

\section{https://pubmed.ncbi.nlm.nih.gov/952626/}

2.DeBaun MR, Jordan LC, King AA, Schatz J, Vichinsky E, Fox CK, et al. American Society of Hematology 2020 guidelines for sickle cell disease: prevention, diagnosis, and treatment of cerebrovascular disease in children and adults. Blood Adv 2020;4:1554-88. https://doi.org/10.1182/bloodadvances.2019001142 
3. Word Health Organisation: WHO. The top 10 causes of death (endereço na Internet). (acessado em 2020). Disponível em: https://www.who.int/news-room/fact-sheets/detail/the-top-10-

causes-of-death

4.Silva RCD, Gurian JG, Curi M, Assis Timpone L, Judice MG, Arantes APF. Funcionalidade e qualidade de vida de indivíduos com AVC pós alta da UTI. Insp Movim Saúde 2019;19:1-18. https://www.inspirar.com.br/wp-content/uploads/2020/02/719.pdf 5. Lee MC, Klassen AC, Resch JA. Respiratory Pattern Disturbances in Ischemic Cerebral Vascular Disease. Stroke 1974;5:612-6. https://doi.org/10.1161/01.str.5.5.612

6.Perlman AL. Disorderes swallowing. In: Tomblin JB, Morris HL, Spriesterbach DC. Disgnosis in Speech - Language Pathology. San Diego: Ed. Singular Publishing Group, 1994; pp361-84.

7.Venketasubramanian N, Seshadri R, Chee N. Vocal cord paresis in acute ischemic stroke. Cerebrovasc Dis 1999;9:157-62. https://doi.org/10.1159/000015947

8.Bassi ERA, Mitre EI, Orate MA, Silva M, Arroyo MAS, Pereira MC. Associação entre disfagia e o topodiagnóstico da lesão encefálica pósacidente vascular encefálico. Rev CEFAC 2004;6:135-42. https://www.fonovim.com.br/arquivos/8d30aeab1b78f3e01454eb799 cd9aa80-Disfagias-no-A.V.C.pdf

9.Terroni LMN, Leite CC, Tinone G, Fráguas R. Depressão pós-AVC: Fatores de risco e terapêutica antidepressiva. Rev Assoc Med Bras 2003;49:450-9. https://doi.org/10.1590/S0104-42302003000400040 10.Girardon-Perlini NMOG, Faro ACM. Cuidar de pessoa incapacitada por acidente vascular cerebral no domicílio: o fazer do cuidador familiar. Rev EsC Enf USP 2005;2:15463. https://doi.org/10.1590/S0080-62342005000200005

11.Arboix A, Marti-Vilalta JL, Garcia JH. Clinical study of 227 patients with lacunar infarcts. Stroke 1990;21:842-7. https://doi.org/10.1161/01.str.21.6.842

12.Darley $\mathrm{FL}$, Aronson $\mathrm{AE}$, Brown JR. Clusters of deviant speech dimensions in the dysarthrias. J Speech Hearing Res 1969;12:462-96. 10.1044/jshr.1203.462

13.Woodson G. Management of Neurologic Disorders of the Larynx. Ann Otol Rhinol Laryngol 2008;117:317-26.

https://doi.org/10.1044/jshr.1203.462

14.Behlau M. Voz: O livro do especialista. São Paulo: Editora Revinter; 2001.

15.Arakawa-Sugeno L. Voz e deglutição de pacientes com e sem mobilidade laríngea após tireoidectomia (Tese). São Paulo: Universidade de São Paulo. 2007.

https://doi.org/10.11606/T.5.2008.tde-28052008-162841

16. Moher D, Shamseer L, Clarke M. Preferred reporting items for systematic review and meta-analysis protocols (PRISMA-P) 2015 statement. Syst Rev 2015;4:1. https://doi.org/10.1186/2046-4053-4$\underline{1}$ 
17.Pithon MM, Sant'anna LIDA, Baião FCS, Santos RL, Coqueiro RS, Maia LC. Assessment of the effectiveness of mouthwashes in reducing cariogenic biofilm in orthodontic patients: a systematic review. J Dentistr 2015;43:297-308.

https://doi.org/10.1016/j.jdent.2014.12.010

18.Godoy JF, Brasolotto AG, Berretin-Félix G, Fernandes AY. Neuroradiology and voice findings in stroke. CoDAS 2014;26:168-74. https://doi.org/10.1590/2317-1782/2014531in

19.Behlau M. Consensus Auditory - Perceptual Evaluation of Voice (CAPE-V), ASHA 2003. Refletindo sobre o novo. Rev Soc Bras Fonoaudiol 2004;9:187-9.

20.Wang YT, Kent RD, Kent JF, Duffy JR, Thomas JE. Acoustic analysis of voice in dysarthria following stroke. Clin Ling Phon 2009;23:335-47. https://doi.org/10.1080/02699200802688604

21.Kay Elemetrics Corp: Multi-Speech and CSL Software: Software Instruction Manual. Lincoln Park: Kay Elemetrics, 2004.

22. Marik PE. Aspiration pneumonitis and aspiration pneumonia. N Engl Med 2001;344:665-7.

https://doi.org/10.1056/NEJM200103013440908

23.Ortiz KZ. Avaliação das Disartrias. In: Ortiz KZ. Distúrbios Neurológicos Adquiridos: Fala e Deglutição. São Paulo: Ed. Manole. 2006; pp84-93.

24.Schalling E, Hammarberg B, Hartelius L. Perceptual and acoustic analysis of speech in individuals with spinocerebellar ataxia (SCA). Logoped Phoniatr 2007;32:31-46.

https://doi.org/10.1080/14015430600789203

25.Ribeiro AF, Ortiz KZ. Perfil populacional de pacientes com disartria atendidos em hospital terciário. Rev Soc Bras Fonoaudiol 2009;14:44653. https://doi.org/10.1590/S1516-80342009000400004

26. Marrara JL. Padrão visual da dinâmica vocal como instrumento para diagnóstico da disfagia em pacientes com alterações neurológicas (Tese). São Paulo: Universidade de São Paulo; 2010. https://doi.org/10.11606/D.18.2010.tde-06052010-160149

27. Maynard MT, Shon RY. Linguagem e funcionalidade de adultos pósAcidente Vascular Encefálico (AVE): avaliação baseada na Classificação Internacional de Funcionalidade, Incapacidade e Saúde (CIF). In: CoDAS; Sociedade Brasileira de Fonoaudiologia; 2007.

28.Urban PP, Rolke R, Wicht S, Keilmann A, Stoeter P, Hopf HC, et al. Left-hemispheric dominance for articulation: a prospective study on acute ischaemic dysarthria at different localizations. Brain 2006;129:767-77. https://doi.org/10.1093/brain/awh708

29.Verdonck-de Leeuw IM, Mahieu HF. Vocal aging and the impact on daily life: a longitudinal study. J Voice 2004;18:193-202. https://doi.org/10.1016/j.jvoice.2003.10.002

30.Ryan WJ, Burk KW. Perceptual and acoustic correlates of aging in the speech of males. J Comm Dis 1974;7:181-92. https://doi.org/10.1016/0021-9924(74)90030-6 
31.Kent RD, Kent JF, Duffy J, Weismer G. The dysarthrias: speechvoice profiles, related dysfunctions, and neuropathology. J Med Speech-lang Pathol 1998;6;165-211.

https://psycnet.apa.org/record/1999-10721-001

32. Kent RD, Kent JF, Duffy JR, Thomas JE, Weismer G, Stuntebeck S. Ataxic dysarthria. J Speech Lang Hearing Res 2000;43:1275-89. https://doi.org/10.1044/jslhr.4305.1275

33.Abberton E. Phonetic considerations in the design of voice assessment material. Logoped Phoniatr Vocol 2005;30:175-80. https://doi.org/10.1080/14015430500298123

34.Andrade LGC. Estudo da Correlação entre Qualidade Vocal e Disfagia Pós-Acidente Vascular Cerebral: Aspectos Acústicos, Fisiológicos e Perceptivos (Dissertação). São Paulo: Pontifícia Universidade Católica de São Paulo. 2004. https://repositorio.pucsp.br/jspui/handle/handle/13822

35.Pereira AC, Brasolotto AG, Berretin-Felix G, Padovani CR. Diadococinesia oral e laríngea em pacientes pós-acidente vascular encefálico. Prófono 2004;16:283-92.

https://pesquisa.bvsalud.org/portal/resource/pt/lil-398004

36.Padovani M, Gielow I, Behlau M. Phonarticulatory diadochokinesis in young and elderly individuals. Arq Neuropsiquiatr 2009;67:58-61. https://doi.org/10.1590/s0004-282x2009000100015

37. Depret MMP. Análise da diadococinesia articulatória e laríngea em indivíduos com e sem transtornos neurológicos (Dissertação). São Paulo: Universidade Federal de São Paulo. 2005. http://repositorio.unifesp.br/handle/11600/20672

38.Urban PP, Wicht S, Vukurevic G, Fitzek C, Fitzek S, Stoeter P, et al. Dysarthria in acute ischemic stroke: lesion topography, clinicoradiologic correlation, and etiology. Neurology 2001;56:1021-7. https://doi.org/10.1212/wnl.56.8.1021

39. Cohen SM, Elackattu A, Noordzij JP, Walsh MJ, Langmore SE. Palliative Treatment of Dysphonia and Dysarthria. Otolaryngol Clin North Am 2009;42:107-21. https://doi.org/10.1016/j.otc.2008.09.010 